\title{
Beiträge zur Kenntnis der Trypsinwirkung.
}

\author{
III. Mitteilung.
}

Die Wirkung des frischen Hundepankreassaftes.

Von

Dr. Karl Mays.

(Der Redaktion zugegangen am 30. Juli 1906.)

Die Entdeckung der Pawlowschen Schule, daß der Pankreassaft in bezug auf seinen Enzymgehalt mit der Nahrung sich ändere, oder daß er sich wenigstens einer bestimmten, längere Zeit dargereichten Nahrung, einem bestimmten Nahrungsregime anpasse, bedarf vielleicht durch die weitere Entdeckung der gleichen Schule, daß ein inaktiver Saft durch eine Substanz, die im Darm, namentlich im Duodenum enthalten ist, die Enterokinase, aktiviert wird, eine gewisse Korrektur, wobei allerdings zu bedenken ist, daß es auch als eine Anpassung an die Nahrung aufzufassen wäre, wenn der Saft zwar nicht Trypsin bei Fleischnahrung enthielte, wohl aber das Zymogen, aus dem dieses hervorgeht. Übrigens fanden Pawlow und seine Schüler gerade bei längerer Fleischkost nur Trypsin im Pankreassaft. ${ }^{1}$ )

Dagegen stellten Délézenne und Frouin ${ }^{2}$ ) und Bayliß und Starling ${ }^{3}$ ) den Satz auf, daß frischer Pankreassaft überhaupt niemals Trypsin enthalte, sondern nur ein anderes, auf genuine Eiweißkörper sehr schwach wirkendes Enzym, das Bayliß und Starling ungerechtfertigter Weise mit dem Erepsin identifizieren. Die apodiktische Behauptung vom Fehlen des Trypsins im Pankreassaft hätte aber doch eine Kritik der früheren Erfahrungen erfordert, bei denen das Gegenteil behauptet wird. Diese Forscher haben zwar gemeint, daß in den Versuchen

1) Vergl. Cohnheim, Biochem. Zentralblatt, 15. Febr. 1903, S. 172.

2) Die Zitate s. b. Cohnheim, a. a. 0.

s) Journ. of Physiol., vol. XXX, p. 61. 
mit Pawlowscher Fistel das minimale Stück Duodenum die Enterokinase geliefert hätte, was indessen noch nicht bewiesen ist. Aber wenn man ihnen auch in dieser Erklärung folgen wollte, so bleiben doch noch viele andere Versuche übrig, wo aus der in den Ausführungsgang eingebundenen Kanüle ein direkt tryptisch wirksamer Saft floß. Außer den alten, aber kaum zu mißdeutenden Angaben von Corvisart und deren Bestätigung durch Kühne hat auch ein Schüler Pawlows, B. B. Kudrewetzky, ${ }^{1}$ ) bei seinen Untersuchungen über den Einfluß der Nervenreizung auf das Pankreassekret, nicht nur bei dieser, sondern auch spontan, wirksamen Saft aus der Kanüle erhalten. Jederrnann weiß heute, daß wenigstens der Hund häufig einen wenig wirksamen Saft sezerniert, und die Tatsache der Aktivierung durch Enterokinase wird in den Vorlesungen über Physiologie demonstriert, aber damit ist nicht gesagt, daß die Abwesenheit von Trypsin im Pankreassafte eine ganz allgemeine Tatsache wäre. Nach Bayliß und Starling wird das Trypsinogen des Pankreassaftes durch gar kein anderes Mittel aktiviert, als durch die Enterokinase, und auch spontan wird kein Trypsin daraus gebildet. Wenn dies richtig ist, so liegt hier ein großer Unterschied zwischen dem Saft und dem Extrakt der Drüse vor, der eine nähere Besprechung erfordert. Daß Enterokinase auch auf das Extrakt der Drüse wirkt, hat Cohnheim²) gezeigt, aber von dem Drüsenextrakt wissen wir seit Heidenhain, daß sein Zymogen sich spontan - sogar bei alkalischer Reaktion - in Enzym verwandelt, wenn auch nicht so rasch wie durch gewisse Mittel, und daß es besonderer Vorsichtsmabregeln bedarf, nämlich sofortigen Übergießens der Pankreasstücke mit Glycerin, ${ }^{3}$ ) um ein direkt unwirksames Extrakt zu erhalten. Daß die spontane Spaltung des Zymogens jedenfalls keine sehr schnelle zu sein braucht, sieht man daraus, daß Vernon ${ }^{4}$ ) aus Rindspankreas, wie es vom Schlachthause

1) Arch. f. Anat. u. Physiol., physiol. Abteil., 1894.

$\left.{ }^{2}\right)$ Arch. des sc. biol. publ. par I'Inst. impér. de méd. expér. à St. Pétersbowrg, Tom. XI, Supplément 1904, p. 112.

8) Hermanns Handbuch d. Physiol., Bd. V, I., S. 188, Anmerking.

4) Journ. of Physiol., vol. XXVI, 1901, p. 405. 
kam, durch geeignete Extraktionsmittel, wie Glycerin oder verdünnten Alkohol, Extrakte erhielt, die erst allmählich ihre tryptische Wirkung entfalteten. Ich habe dagegen, wenn ich Rindspankreas, wie sie vom Schlachthause kommen, mit Wasser oder indifferenten Salzlösungen ansetzte, nach wenigen Tagen Extrakte von maximaler Wirkung erhalten ${ }^{1}$ ) und auch schon in kürzerer Zeit recht erhebliche Wirkung beobachtet. Es ist aber damit nicht gesagt, daß dies immer so sein müsse, und ich kann hier nur wieder darauf hinweisen, daß beim Pankreas immer Verschiedenheiten erwariet werden müszen; so hat z. B. Sachs ${ }^{2}$ ) beobachtet, daß in einem Falle das Extrakt eines solchen Pankreas nach 17 stündiger Extraktion eine Fibrinflocke in 6 Stunden nicht angriff, während ein anderes (sogar etwas verdünnteres) nach 18 stündiger Extraktion eine Fibrinflocke in einer Stunde löste.

Wieder anders scheint die Sache beim Katzenpankreas zu liegen. Diese Drüsen sind im Anfang, auch bei Extraktion mit Wasser, meist wenig oder direkt gar nicht wirksam; auch hier habe ich zwar ein spontanes Wirksamerwerden beobachtet, aber die Wirksamkeit war oder wurde gewöhnlich längst nicht so groß wie beim Rindspankreasextrakt. Ausnahmsweise habe ich aber auch aus frischem Katzenpankreas ein direkt sehr gut wirkendes Extrakt erhalten.

Bei Hunden erhielt Kühne, ${ }^{3}$ ) wenn er sie in der sechsten Stunde nach der letztẹ Fütterung schlachtete, meist sehr gut wirksame Extrakte und bemerkt, daß unwirksame Extrakte überhaupt selten sind; auch nach sechs Tagen Hunger lieferten Hunde noch trefflich wirkende Extrakte; nur bei lange schlecht gehaltenen und durch Vivisektion herabgekommenen Tieren stieß er gelegentlich auf unwirksame Infuse, die aber durch einige Stunden langes Behandeln in der Wärme unter leichtem Ansäuern wirksam wurden, und er hat schon damals den Satz ausgesprochen: Nach meinen Beobachtungen muß es

1) Diese Zeitschrift, Bd. XXXVIII, S. 428.

2) Inaugural-Dissert. Heidelberg 1905, J. Hörning, und Diese Zeitschrift, Bd. XLVI, S. 337.

8) Virchows Archiv, Bd. XXXIX, S. 130, 1867. 
scheinen, als ob die unwirksame Drüse eine Substanz enthalte, welche erst bei Digestion mit Säuren zerfalle unter Abspaltung des Fermentes». Obwohl Heidenhain ${ }^{1}$ ) hierüber bemerkt: «Was Kühne für eine Eigentümlichkeit nur eines besonderen Drüsenzustandes hält, hat Allgemeingültigkeit für jedes Pankreas », so möchte ich doch die Möglichkeit betonen, daß hier wieder etwas anderes vorliegt, indem diese Infuse Kühnes nicht spontan Enzym lieferten, sondern nur bei Behandlung mit Säuren.

Den Unterschied zwischen Pankreasextrakt und Pankreassaft hat schon Kühne zu einer besonderen Lehre entwickelt. ${ }^{2}$ ) Auch er nahm, gestützt auf das häufige Vorkommen eines direkt wenig wirksamen Saftes beim Hunde, an, daß dieser eine Vorstufe des Trypsins enthalte; aber er unterscheidet zwischen einem $\mathrm{Zy}$ mogen des Extraktes und einem Trypsinogen des Saftes. Die Unterschiede bestehen nach ihm darin, daß Zymogen nur durch Säuren gespalten werde (bei der spontanen Aktivierung machte er auch die Säuerung verantwortlich; dies stimmt aber nicht mit der Beobachtung Heidenhains, daß diese auch in alkalischer Lösung stattfindet) und durch Alkohol bei Temperaturen, die wesentlich über $0^{0} \mathrm{C}$. liegen (jedoch schon bei Zimmertemperatur). Bereite man dagegen aus frischen Drüsen ein eiskaltes Extrakt, so könne man mit kaltem Alkohol daraus das Zymogen fällen, das, gelöst, unwirksam sei und nur durch Säuren gespalten werde. Das Trypsinogen werde dagegen auch durch kalten Alkohol gespalten, da die Lösung des dadurch entstandenen Niederschlages sofort wirksam sei; außerdem werde es auch gespalten in alkalischer Lösung durch Sauerstoff, was bei Zymogen nicht der Fall sei. (Im Gegensatz dazu nimmt Heidenhain nach den Arbeiten Podolinskis an, daß der Sauerstoff zu den Mitteln gehöre, die das Zymogen spalten.) Kühne meint, das Trypsinogen werde aus dem noch komplizierten Zymogen abgespalten, und aus ihm erst gehe das Trypsin hervor, nicht direkt aus dem Zymogen, wie Heidenhain annimmt. Diese Untersuchungen Kühnes werden noch eingehende Be-

1) Pflügers Arch., Bd. X, S. 587.

2) Verhandl. d. naturh.-med. Vereins zu Heidelberg, N. F., I. Heft 3 u. 4 ; z. Teil auch nicht publiziert. 
arbeitung bedürfen; daß aber zwischen dem Extrakt und dem Saft ein Unterschied zu machen ist, geht auch aus den neueren Versuchen von E. Fischer und P. Bergell hervor, $\left.{ }^{1}\right)$ bei denen gefunden wurde, daß gewisse Peptide, z. B. Leucyl-Alanin, durch ein Extrakt der Pankreasdrüse partiell gespalten werden, ${ }^{2}$ ) während sie sowohl für den durch Darmsaft aktivierten als auch für «spontan aktiv " gewordenen Saft ganz unzugänglich sind.

Da diese Forscher von «spontan aktiv' gewordenem Saft sprechen, steht auch ihre Ansicht in Widerspruch zu den Angaben von Délézenne und Frouin und von Bayliß und Starling.

Da mir mehrere Proben von frisch sezerniertem Pankreassaft des Hundes zu Gebote standen, habe ich dieselben benutzt, um die Resultate der genannten Forscher zu kontrollieren.

Versuch 1.

Von Herrn Kollegen Plenge erhielt ich einige Kubikzentimeter Pankreassaft vom Hund, den er aus der Kanüle gesammelt hatte, nachdem etwas verdünnte Salzsäure ins Duodenum gebracht worden war. Der Saft ist ganz klar und reagiert kräftig alkalisch.

Damit wurden folgende Versuche angestellt:

1. Auf Fibrin war der Saft von geringer Wirkung; in einer Stunde war an einer Flocke frischem Rindsfibrin bei $40^{\circ} \mathrm{C}$. keine Veränderung zu bemerken. Nach einigen Stunden war die Flocke teilweise und am nächsten Morgen ganz gelöst.

2. $2 \mathrm{ccm}$ Saft wurden mit $1 / \mathrm{s} \mathrm{ccm}$ Peptonlösung (Plasmonpepton, welches Cohnheim verwendet hat) ${ }^{3}$ ) versetzt und warm gestellt. Nach 2 Tagen war noch eine sehr gute Biuretreaktion nach dem Auskoagulieren der Probe $z u$ konstatieren.

3. Nachdem der Saft einen Monat unter Toluol gestanden hatte, wobei sich Tyrosin ausgeschieden hatte, aber keine Spur von Fäulnis zu erkennen war, griff er eine Flocke gekochtes Fibrin über Nacht bei $40^{\circ} \mathrm{C}$. so an, daß sie beim Schütteln in sehr kleine Partikel zerfiel. Ebenso wurde in der gleichen Zeit gekochtes Eierweiß bis auf kleine Stücke und nach weiteren 24 Stunden klar gelöst.

Ich habe erst nach Beendiguug dieses Versuches gesehen, daß Bayliß und Starling die Nichtlöslichkeit von koaguliertem Albumin

:) Diese Zeitschrift, Bd. XLVI, S. 52.

$\left.{ }^{2}\right)$ Ber. d. Deutsch. chem. Gesellsch., Bd. XXXVII, S. 3103.

3) Diese Zeitschrift, Bd. XXXV, S. 410. 
nur für Versuche bei Zimmertemperatur behaupten, und deshalb den folgenden Versuch viel später, mit dem aber jetzt immer noch tadellos konservierten Safte angestellt.

4. Nachdem der Saft 4 Monate gestanden hatte, wurde eine Probe desselben mit gekochten Eierweißwürfeln bei Zimmertemperatur stehen gelassen und daneben eine gleiche Probe, bei der aber der Saft gekocht war. Schon am folgenden Tag zeigten sich äußerlich Verschiedenheiten. Während die Eiweißwürfel im gekochten Saft unverändert erschienen, waren die Ränder der in dem wirksamen Saft befindlichen abgerundet und durchsichtig und die Würfel zerfielen beim Schütteln (das auf die in dem gekochten Safte ganz ohne Einfluß war) in feine Partikel. Nach weiteren 10 Tagen war das Eierweiß im wirksamen Safte klar gelöst, im gekochten unverändert.

Aus diesen Versuchen geht hervor, daß frischer Hundepankreassaft mindestens keine hervorragende ereptische Eigenschaft besitzt, wenigstens nicht immer besitzt, da zugesetztes Pepton in 2 Tagen bei $40^{\circ} \mathrm{C}$. nicht wesentlich zerstört wurde; ferner, daß der Saft in seiner proteolytischen Wirkung sich wie eine schwache Trypsinlösung verhält auch in bezug auf Lösung koagulierten Eiweißes bei Zimmertemperatur. Allerdings war letzteres erst konstatiert worden, nachdem der Saft längere Zeit gestanden hatte und vielleicht dies Vermögen erst erlangt hatte; dann läge aber gegen die Anschauung von Bayli $\beta$ und Starling mindestens eine spontane Trypsinbildung vor.

\section{Versuch 2 .}

Herr Frouin war einige Zeit Gast des hiesigen Instituts. Von ihm erhielt ich $16 \mathrm{ccm}$ Hundepankreassaft, den er aus der Kanüle nach Sekretininjektion erhalten hatte. Der Saft war nicht ganz klar und wurde aus äußeren Gründen nicht sofort untersucht, aber sogleich toluolisiert.

Am folgenden Tag wurde der Saft filtriert. Er lief nicht ganz klar durchs Filter. Irgend welche Bakterien waren, wie die mikroskopische Untersuchung erwies, nicht darin zu entdecken. Mit diesem Safte wurden folgende Versuche angestellt:

1. Auf eine Flocke Fibrin wirkte der Saft (am 2. Tage) bei $40^{\circ} \mathrm{C}$. folgendermaßen :

20 Min. = 0; 30 Min.: bg. Z. ; 40 Min. : mäß. R.; 70 Min. : ger. R.; 85 Min.: min. R.; nachmittags : gelöst.

2. Am 3. Tage wurde eine Probe des Saftes mit Würfeln von ganz frisch abgekochtem Hühnereierweiß bei Zimmertemperatur hingestellt. Am folgenden Tage erschienen die Würfel verkleinert und zeigten beim

Hoppe-Seyler's Zeitschrift f. physiol. Chemie. XLIX. 
Schütteln starken Zerfall; nach 2 weiteren Tagen waren sie ganz gelöst und in der Probe fand sich Tyrosin ausgeschieden, was in dem Safte allein bis dahin nicht der Fall war.

Nach weiteren 20 Tagen wurde dies Gemisch filtriert und mit Kochsalz und Essigsäure auskoaguliert. Das Filtrat gab eine sehr intensive, schön purpurne Biuretreaktion.

3. Nachdem der Saft 23 Tage gestanden hatte, war seine Wirkung auf frisches Fibrin entschieden besser.

15 Min.: bg. Z.; 30 Min.: kl. R.

4. Zu dieser Zeit wurden zwei Proben des Saftes mit etwas Extrakt aus je einem Katzendarm (a und b) versetzt. Dadurch wurde seine Wirkung nicht gebessert.

5. Nach 5 Monaten löste der Saft eine frische Fibrinflocke noch auf, aber in längerer Zeit, so daß er in dieser Beziehung entschieden wieder geschwächt war; nämlich :

30 Min.: ?; 60 Min.: zieml. Z.; 90 Min.: ger. R.

Auch bei diesem Versuch, in welchem der Saft in der gleichen Weise gewonnen wurde, wie dies von Bayliß und Starling geschah, zeigte sich, und zwar sehr bald, eine entschiedene Wirkung auf gekochtes Eierweiß bei Zimmertemperatur. Da in der Lösung dieses Eiweißes nach 25 Tagen die Biuretreaktion noch so intensiv war, konnte also auch hier von einer wesentlichen ereptischen Wirkung nicht die Rede sein. Der Saft wirkte mit der Zeit spontan besser fibrinlösend, büßte aber im weiteren Verlaufe an dieser Kraft wieder ein, wie dies auch bei Trypsinlösungen der Fall ist. Eine Besserung des Saftes zu einer Zeit, wo er schon an und für sich besser wirkte, durch Enterokinase, die man in den Darmextrakten der Katze erwarten durfte, war aber auch nicht zu konstatieren.

\section{Versuch 3.}

Ebenfalls von Herrn Frouin erhielt ich $25 \mathrm{ccm}$ ganz klaren Hundepankreassaft, den er in der gleichen Weise nach Sekretininjektion erhalten hatte.

1. Derselbe wirkte sofort recht schwach auf Fibrin, da eine frische Flocke in 30 Min. keine Spur von Zerfall zeigte. Über Nacht wurde sie bei Zimmertemperatur auch nicht angegriffen. Nun wieder auf $40^{\circ} \mathrm{C}$. gebracht, zeigte die Flocke nach einigen Stunden Zerfall und war am nächsten Tage, nachdem die Probe über Nacht wieder bei Zimmertemperatur gestanden hatte, gelöst.

2. Sofort nachdem ich den Saft erhalten hatte, setzte ich eine Probe davon mit einigen Würfeln gekochtem Eierweiß bei Zimmer- 
temperatur an. Zwei Tage lang zeigte dasselbe keinen Zerfall. Nach weiteren 2 Tagen zerfiel es bei starkem Schütteln. Eine Probe dieses Gemisches gab nach der Filtration beim Koagulieren ein mäßiges Koagulum und im Filtrat davon war keine Biuretreaktion zu erkennen.

Wir haben also hier einen sofort sehr wenig wirksamen Saft vor uns, wie dies schon aus der sehr geringen Wirkung auf Fibrin hervorgeht. Da diese aber so gering ist, ist die geringe Wirkung auf koaguliertes Eierweiß bei Zimmertemperatur nicht auffallend, aber das Vorhandensein dieser Wirkung bedeutungsvoll, da sich eben auch hier kein Unterschied gegen Trypsin konstatieren läßt. Merkwürdig ist nur der negative Ausfall der Biuretreaktion. Da nicht alles Eiweiß gelöst war und etwas gelöstes bei der Koagulation ausfiel, so könnte man annehmen, daß die ganze Wirkung des Saftes eben nur in einer Lösung eines Teiles des Eiweißes bestanden habe und es noch gar nicht zur Bildung von unkoagulablen Körpern gekommen sei. Da aber, wie wir gleich sehen werden, der Saft allein sein eigenes Pepton nur schwer bewältigt, so hätte doch das aus ihm selbst stammende Pepton sich hier erkennen lassen müssen. Eine zureichende Errklärung hierfür finde ich nicht.

3. Es wurde am vierten Tage folgender Parallelversuch gemacht: 1. Eine Probe des Saftes allein, 2. 2,2 ccm Saft $+0,8 \mathrm{ccm}$ einer Peptonlösung (auf deren Gewinnung ich unten zurückkommen werde) wurden in den Wärmeschrank gebracht. Nach 3 Tagen war in beiden Proben noch eine sehr deutliche Biuretreaktion (nach dem Koagulieren) zu erkennen und zwar war sie in beiden Proben auffallend gleich.

Von da an standen die beiden Gemische bei Zimmertemperatur. Nach neun Tagen wurden die Reste koaguliert, wobei nur eine ganz geringe Trübung entstand. In den Filtraten war noch Biuretreaktion zu erkennen; auffallenderweise fiel sio in der Probe, in der das Pepton zugesetzt war, deutlich geringer aus als in dem Saft allein.

Der Versuch war angestellt worden, um zu sehen, ob in ganz frischem, nicht aktiviertem Hundepankreassaft sich etwa Erepsin fände. Eine sehr energische Wirkung auf Pepton war jedoch sicher nicht vorhanden. Nach längerer Zeit wurde aber doch ziemlich viel Pepton zerstört; es scheint aber in dem Saft selbst ein Körper zu sein, der ein recht schwer angreifbares Pepton liefert, wodurch das Urteil über die Spaltung des zugesetzten Peptons erschwert wird. 
Ich versuchte nun, ob dieser recht wenig wirksame Saft durch geeignete Mittel besser wirksam gemacht werden könne.

4. Eine Probe des Saftes wurde am 3. Tage nach der Gewinnung einige Stunden auf $40^{\circ} \mathrm{C}$. erhalten, dann abgekühlt und mit gekochtem Eierweiß bei Zimmertemperatur stehen gelassen. Am folgenden Tage waren die Eiweißwürfel auffallend weich und leicht zerdrückbar, wurden aber dann in längerer Zeit kaum weiter verändert.

Wenn hier auch bälder als bei ganz frischem Saft eine Erweichung des koagulierten Eierweißes eingetreten war, so war doch die weitere Wirkung so gering, daß man den Saft durch die Erwärmung auf $40^{\circ}$ C. als nicht viel gebessert betrachten durfte.

5. $1 \mathrm{ccm}$ Saft wurde am 4. Tage mit 0,6 ccm Plasmonpepton versetzt. Diese Mischung hatte in 30 Minuten bei $40^{\circ}$ C. keine Wirkung auf eine frische Fibrinflocke. Nachdem die Probe über Nacht bei Zimmertemperatur gestanden hatte, war die Flocke gelöst.

Diese Wirkung auf Fibrin ist entschieden besser als die des frischen Saftes. Ich glaubte zuerst eine Wirkung des Peptons erkennen $\mathrm{zu}$ sollen, welches ja auch, wie ich früher beschrieben habe, wenig wirksame Katzenpankreasextrakte bessert; es ist aber in diesem Falle nicht ausgeschlossen, daß es sich um eine spontane Aktivierung des Pankreassaftes handelte, da er est am 4. Tage zu dieser Probe benutzt wurde.

Nach Kühne soll Trypsinogen durch Alkohol gespalten werden und es war zu versuchen, ob auf diesem Wege aus dem Safte eine wirksamere Lösung zu gewinnen war. Es wurden deshalb

6. am folgenden Tage nach der Gewinnung $5 \mathrm{ccm}$ Saft mit viel Alkohol versetzt. Merkwürdigerweise entstand dabei nur eine geringe flockige Făllung. Am Nachmittage saß diese am Boden, wurde auf ein Filter gesammelt und dies mit $5 \mathrm{ccm} 0,5 \%$ iger Natriumbicarbonatlösung extrahiert. Diese Lösung wirkte in $30 \mathrm{Min}$. bei $40^{\circ} \mathrm{C}$. nicht auf frisches Fibrin. Über Nacht stand die Probe bei Zimmertemperatur. Am folgenden Tage war die Fibrinflocke auch noch nicht angegriffen, am dritten Tage war sie gelöst. Einige jetzt in die Probe eingebrachte Würfel von gekochtem Eierweiß wurden bei Zimmertemperatur in einem Tage nicht verändert, nach weiteren 2 Tagen waren sie beim Zerdrücken auffallend weich, wurden aber dann nicht weiter verändert.

Auch dieser Versuch, Trypsinogen in Trypsin zu verwandeln, war also vergeblich; aber dennoch enthielt dieser 
Saft die Vorstufe des Enzyms und zwar in reichlicher Menge, wie der folgende Versuch lehrt:

7. Am dritten Tage nach der Gewinnung des Saftes wurden $3 \mathrm{ccm}$ mit $1 \mathrm{ccm}$ des S. 194 erwähnten Extraktes aus Katzendarm b versetzt, das allein in 45 Minuten keine Wirkung auf frisches Fibrin zeigte. Diese Mischung wirkte sehr gut auf Fibrin; nämlich auf eine frische Flocke:

5 Min.: zieml. Z.; 10 Min.: ger. R.; 15 Min.: gelöst.

Es war von Interesse, zu untersuchen, ob dieser gut wirkende aktivierte Hundepankreassaft eine besonders gute ereptische Wirkung habe; deshalb wurde in die Probe, in der die Flocke Fibrin gelöst war, etwas mehr Fibrin hineingebracht und warm gestellt. Am folgenden Tag entstand beim Koagulieren ein geringes flockiges Koagulum; im Filtrat davon war die Biuretreaktion noch sehr stark. Da eine Probe dieses Filtrates mit Ammonsulfat kaum eine Trübung gab, war hier vorzugsweise echtes Pepton gebildet.

Fine besonders große ereptische Wirkung hat also der aktivierte Saft auch nicht, obwohl er Fibrin rasch löst und rasch in echtes Pepton verwandelt.

Dies Verdauungsgemisch wurde nun gekocht und stellte so die Peptonlösung dar, die zu dem oben unter 3. S. 195 erwähnten Versuch benutzt wurde.

Wie wir gesehen haben, haben E. Fischer und O. Bergell gefunden, daß, auch durch Enterokinase, aktivierter Hundepankreassaft Peptide nicht angreift, die durch ein Extrakt der Drüse gespalten werden. Da nun verschiedene ihrer Peptide die Biuretreaktion geben, so müßte, wenn diese bei der Verdauung auftreten, ein Eiweißverdauungsversuch entscheiden, ob auch diese Körper wie das Leucyl-Alanin von aktiviertem Pankreassaft nicht angegriffen werden, oder ob eine Verdauung mit solchem schließlich zu nur abiureten Produkten führte. Wenn ich auch gezeigt habe, daß eine besonders rasche peptonspaltende Wirkung auch bei dem aktivierten Safte nicht nachweisbar ist, so konnte sie doch mit der Zeit eine vollständige sein. Ich habe einen solchen Versuch angestellt, der aber leider wegen der Knappheit des Materials nur unvollständig sein konnte. 


\section{Versuch $\{$.}

Herr Kollege Plenge gab mir $10 \mathrm{ccm}$ ganz klaren Hundepankreassaft, den er nach Sekretininjektion aus der Kanüle erhalten hatte. Zunächst wurde seine verdauende Wirkung geprüft.

1. Es wurden deshalb angesetzt bei Zimmertemperatur:

a) Saft + Fibrin,

b) Saft + ganz frisch koaguliertes Eierweiß,

c) Kontrollprobe: Saft gekocht + koaguliertes Eierweiß.

Nach 2 Tagen schien das Fibrin teilweise erhalten, zerging aber beim Schütteln in feinste Partikel. Auch das Eierweiß in b) war scheinbar unverändert, zerfiel aber beim Zerdrücken leicht zu einem feinen Detritus. Nach weiteren neun Tagen zerfielen die Eiweißwürfel in b) leicht beim Schütteln und nach weiteren 15 Tagen war nichts mehr davon zu sehen und die ganze Probe in eine milchige Flüssigkeit verwandelt (zu der milchigen Beschaffenheit solcher Verdauungsgemische trägt eine Emulsion des Toluols wesentlich bei), während die Eiweißwürfel in der Kontrollprobe c) ganz unverändert waren.

Von dem gleichen Hunde wurde ein Stück Darm (da das Duodenum zu anderen Zwecken gebraucht war, mußte ich ein Stück des Jejunum nehmen) mit alkalischer Kochsalzlösung extrahiert.

Es wurden nun angesetzt:

a) $4,5 \mathrm{ccm}$ des Saftes +2 Tropfen des Darmextraktes $+1 \mathrm{ccm}$ Fibrinpepton (das Pepton war erhalten durch Einlegen von gekochtem Fibrin in Rindspankreasextrakt, wobei es sich mit Trypsin belud und Digestion dieses Fibrins in alkalischer Lösung, die, nachdem reichlich Pepton in Lösung war, angesäuert und abgekocht wurde).

b) Eine Probe des gleichen Gemisches + eine Flocke frisches Rindsfibrin.

c) Der Rest des Saftes $=2 \mathrm{ccm}$ wurde gekocht und wieder abgekühlt, dazu ein Tropfen des Darmextraktes gesetzt und $1 / 2 \mathrm{ccm}$ des gleichen Peptons.

Die 3 Proben wurden warm gestellt.

Das Fibrin in b) zeigte nach einigen Stunden Zerfall und war am anderen Morgen gelöst.

Nach 7 Tagen wurden a) und c) gekocht, wobei nur ganz geringe Trübung eintrat, und die Biuretprobe gemacht. Leider hatte ich kein Material mehr, um eine frisch bereitete Probe eines solchen Gemisches mit den digerierten zu vergleichen, und kann deshalb nur sagen, daß die Biuretprobe zwar noch vorhanden war, aber so schwach ausfiel, daß eine Zerstörung von Pepton kaum zu bezweifeln war. Die Farbe der Biuretreaktion war aber in beiden Proben nahezu gleich. Die Kontrollprobe c) war damit erschöpft; a) blieb noch weitere 7 Tage im Wärmeschrank. Nun fiel die Biuretreaktion nach dem Kochen und Filtrieren 
vollständig negativ aus und bei Zusatz von 10 Tropfen Kupferlösung erschien die Probe nur blaßblau.

Dieser Saft war von mäßiger, aber deutlicher lösender Wirkung auf Eiweißkörper. Eine sehr wesentliche Besserung durch Darm- (allerdings Jejunum-) Extrakt war nicht zu konstatieren. Dagegen hat so behandelter Saft Pepton nach einiger Zeit vollständig zerstört. Da aber eine Kontrollprobe mit gekochtem Saft und wirksamem Darmextrakt auch Anzeichen von Peptonzerlegung gab, ist dieses Resultat mit Vorsicht aufzunehmen. Auffallend ist, eine wie geringe Menge des Darmextraktes (1 Tropfen auf im ganzen $2^{1} / 2 \mathrm{ccm}$ ) genügte, um diese Peptonspaltung $\mathrm{zu}$ bewirken, aber es bedurfte dazu doch längere Zeit. Man könnte denken, daß eine Peptonzerstörung ohne Enzyme vorläge; ich habe indessen häufiger Peptonlösungen längere Zeit im Wärmeschrank stehen lassen und nie eine $\mathrm{Ab}$ nahme der Biuretreaktion bemerkt. Vernon, ${ }^{1}$ ) der eine solche spontane Zersetzung in Sodalösung beobachtet haben will, setzte doch selbst einigen Zweifel in die Sicherheit der Beobachtung. Liegt aber hier eine enzymatische Wirkung vor, so ist auch der Versuch, in dem der Pankreassaft wirksam war, in welchem schließlich das ganze Pepton bewältigt wurde, noch nicht beweisend. Allerdings wurde dieser Saft durch das Darmextrakt in bezug auf seine lösende Wirkung wenig gebessert, so daß in diesem Extrakt vielleicht nnr wenig Kinase vorhanden war, aber man hat eben bei Darmextrakten immer mit zwei Dingen zu rechnen: mit Enterokinase und mit Erepsin. Die Versuche mit «spontan aktiv gewordenemPankreassaft wären beweisender.

Überblicken wir diese Versuche, so sehen wir daraus, daß der Hundepankreassaft, wie er aus der Kanüle der temporären Fistel fließt, häufig direkt von sehr geringer proteolytischer Wirksamkeit ist; aber auch bei solchen Säften gibt es schon ein gewisses Mehr oder Weniger dieser Wirkung. Ein Saft, der die Eigenschaft besäße, wie sie Bayliß und Starling beschreiben, der nur Fibrin, nicht aber koaguliertes Eiweiß löse, ist mir nicht vorgekommen, womit aber nicht gesagt sein soll, daß es nicht auch solche Säfte gäbe oder vielleicht sogar direkt

1) Journ. of Physiol., Bd. XXX, S. 337. 
ganz unwirksame; aber ich kann daraus nicht auf ein besonderes Enzym des Pankreassaftes schließen, sondern würde darin bei den schwach wirkenden nur eine sehr schwache Trypsinwirkung erblicken, die es eben nicht mehr zu der Lösung des schwerer angreifbaren Eierweißes brächte. Dem Versuch von Bayliß und Starling, daß ein auf Fibrin auch bei $40^{\circ}$ schwach wirksames Pankreasextrakt doch noch auf Gelatine lösend wirke und sich dadurch als tryptisch dokumentiere, kann ich nur meine Erfahrungen gegenüber halten, daß auf Fibrin auch sehr schwach wirkender Hundepankreassaft eben doch auch deutlich, wenn auch langsam auf koaguliertes Eierweiß wirkt.

Wenn Bayli $\beta$ und Starling sagen, daß das Trypsinogen, das ja zweifellos in diesen schwach wirkenden Säften neben den geringen Trypsinmengen vorhanden ist, nur durch Enterokinase aktiviert werde, so kann ich nicht unerwähnt lassen, daß ich doch auch durch andere Mittel oder spontan eine, wenn auch nicht sehr bedeutende Besserung der tryptischen Wirkung des Saftes gesehen habe. Zweifellos steht jedoch die Enterokinase diesen Mitteln weit voran. Es ist auch die Möglichkeit nicht ausgeschlossen, daß im Pankreassafte verschiedene Vorstufen des Trypsins vorkommen, von denen vielleicht eine nur durch Enterokinase gespalten wird, andere auch in anderer Weise. Ich darf dabei vielleicht an die Verschiedenheiten erinnern, die in Kühnes und Heidenhains Erfahrungen über die Aktivierung der Drüsenextrakte liegen.

Wir haben keinen Grund gefunden, bei schwach eiweißlösenden Säften ein anderes Enzym als Trypsin anzunehmen, das wohl 'gelegentlich in so geringer Menge vorhanden sein kann, daß das schwer angreifbare koagulierte Eiweiß nicht mehr bei Zimmertemperatur angegriffen wird, oder das auch vielleicht ganz fehlen kann, ohne daß dieses Fehlen von Trypsin in frischem Safte prinzipiell wäre. Dann ist aber nicht einzusehen, warum auf der anderen Seite unter geeigneten Bedingungen nicht auch direkt sehr gut tryptisch wirkende Säfte sezerniert werden sollten, wie es frühere Beobachter angegeben haben, und der Satz von Délézenne und Frouin und von 
Bayliß und Starling, daß der Hundepankreassaft nie Trypsin enthalte, kann nicht zu Recht bestehen.

In bezug auf die Frage, ob ein Pankreasdrüsenextrakt Erepsin enthalte, und in Anbetracht der namentlich von Fischer und Bergell beobachteten Verschiedenheit von Extrakt und Saft, war von Interesse $\mathrm{zu}$ sehen, wie sich letzterer in bezug auf ereptische Wirkung verhielte. Diese Versuche sind noch auf nicht aktivierte Säfte auszudehnen in der Art, daß diese längere Zeit auf Pepton einwirken gelassen werden. In kürzerer Zeit ist jedenfalls keine besonders große ereptische Wirkung, weder bei nicht aktiviertem noch bei durch Darmextrakte aktiviertem Safte vorhanden. Längere Zeit mit durch Darmsaft aktiviertem Pankreassaft angestellte Versuche müssen die ereptische Wirkung des Darmextraktes selbst in Rechnung ziehen. 